\title{
Energy Spectra of Cascade Electrons and Photons ${ }^{2}$
}

\author{
Charles A. Olson ${ }^{2}$ and Lewis V. Spencer
}

\begin{abstract}
The equations for the energy spectra of electrons and photons in a cascade shower are written in a form suitable for numerical applications with accurate cross sections. Trial calculations were carried out to check the feasibility of a step-by-step numerical integration procedure similar to that used successfully at low energies in noncascade problems. Numerical results were obtained for lead by using a monoenergetic source of electrons at $360 m c^{2}$ and extreme relativistic cross-sectional forms, which assume complete screening. The method was designed to permit the determination of cascade-shower spectra, spatial distributions, and directional distributions that are exact in the sense that the main limiting factor is the accuracy with which the cross sections are known. The numerical results of the trial calculations were found to agree with previously published results based on more restrictive methods of analysis.
\end{abstract}

\section{Introduction}

High-energy electrons and photons dissipate their energy by generating secondary electrons and photons. The complex radiation arising in this manner from a high-energy electron or photon source is called a cascade shower. Most attempts to describe this phenomenon have used approximations that are valid only at very high energies, i. e., in the extreme relativistic range; and the results have only limited applicability in a broad range of lower energies where the cascade phenomenon is still of importance. At these lower energies realistic calculations must be largely numerical because the accurate cross-sectional forms are too complicated to permit analytic transformations.

In this paper a calculational procedure which applies without restrictions on cross-sectional forms, and which has been used in noncascade problems, is adapted to the cascade situation. It should provide an adequate way of describing cascade-shower spectra in any energy range. Because the equations are part of a linked set of moment equations, which describe also spatial and directional distributions, and possibly fluctuations, the procedure in this paper may eventually form the basis for a more complete description of many aspects of the cascade problem.

The equations are applied, in a trial calculation, to the special case of a monoenergetic electron source. Calculations for an electron source are more important than for a photon source for the following reason: If a photon source is assumed, it is possible to calculate the resulting photon spectrum at all energies. This type of calculation has been done many times and offers no difficulty (for an example, see reference [1]). ${ }^{3}$ One can then determine by simple quadrature the electron spectrum generated by this photon spectrum. The whole cascade spectrum of electrons and photons, except for the initial photon component already determined, can then be determined by appropriate superposition of solutions for monoenergetic electron sources. Thus, calculations for a monoenergetic electron source demonstrate that the main difficulty blocking a complete numerical analysis of the cascade problems is removed.

For the initial calculation, extreme relativistic cross-sectional forms that assume complete screening are used. They include the same types of singularity as the exact cross sections and therefore provide a meaningful trial run. Also they are quite similar to the cross-sectional forms used in earlier applications of conventional cascade theory; so they make possible a direct check with previously published results.

\footnotetext{
1 This work was supported by the Office of Naval Research and the Atomic Energy Commission.

2 Submitted to the University of Maryland in partial fulfillment of the requirements for the M. S. degree.

3 Figures in brackets indicate theli ${ }^{*}$ ture references at the end of this paper.
} 


\section{Interactions Involved in Cascade Showers}

The two types of interactions that predominate in the development of cascade showers are bremsstrahlung and pair production. In a bremsstrahlung interaction a high-energy electron or positron, under the influence of a nuclear or electronic field, generates a photon. In pair production a high-energy photon in the electromagnetic field of a nucleus or electron gives up all its energy to generate an electron and a positron. The combination of these two processes results in the creation of photons, electrons, and positrons in increasing numbers at lower energies.

At all energies below the extreme relativistic range, there are additional effects that influence the development of the showers. There are energy losses due to atomic interactions that slow down the electrons more or less continuously along their path. Some of these interactions result in secondary electrons with high energies, i. e., knock-on electrons. Compton scattering also contributes some high-energy electrons to the shower, but at low energies it is of primary importance through its influence on the photon spectrum. The photoelectric effect and photonuclear interactions have cross sections that are much smaller than the other interactions at all cascade-shower energies, though the former is of importance below shower energies.

\section{Discussion of Earlier Work}

An excellent discussion of the lines along which the mathematical description of the cascade phenomenon has been developed can be found in a book by B. Rossi [2]. Because the procedural methods of this paper are directed mainly toward a solution of the cascade problem at low energies where accurate cross-sectional forms are needed, comments here on earlier work will be restricted to developments pertinent to these lower energies.

The first attempt to describe a low-energy cascade spectrum was made by Arley [3]. He neglected interactions that slow down the electrons, i. e., ionization losses, above the critical energy; ${ }^{4}$ and he further neglected bremsstrahlung and the generation of secondary electrons by all processes at energies below the critical energy. His results as compared with more recent calculations are low by a factor of 3 or 4 .

Tamm and Belenky [4] have obtained a much more accurate solution by an analytic method, using asymptotic cross-sectional forms for bremsstrahlung and pair production, assuming continuous ionization loss and neglecting the Compton effect and knock-on electrons. Their solution has proved to be more accurate than was at first expected, considering the approximations. Friedman [5] has discussed their solution at length and computed a first correction term. This correction term never exceeded 4.5 percent of the original solution in the case that Friedman considered.

Perhaps the most satisfactory treatment of the energy-spectra problem for low energies has been given by Richards and Nordheim [6]. They have used reasonably accurate representations for all important interactions, but they treat small radiation and ionization losses as continuous, thus incurring an error of up to 8 percent, according to their own estimates. They obtain results by numerical integration for energies from $1 / 2$ to 10 times the critical energy. Their results are not absolute and do not describe the spectra near the initial energy. To obtain more complete spectra, it is necessary to normalize their equations to a solution valid at high energies, and they have used the results of Rossi and Greisen [7] for this purpose.

All these calculations are difficult to generalize to a more accurate treatment of all the different interactions, and hence there is a need for a new approach that will permit use of arbitrarily accurate cross sections.

\section{Analytic Treatment of the Problem}

In this section equations will be derived for the energy spectra of electrons and photons. No distinction is made between positrons and electrons, the two being considered as having

${ }_{4}^{4}$ The critical energy in a given material is the energy at which the radiation losses of an electron equal the ionization losses. 
identical cross sections and combining to make up an "electron" spectrum. The differential energy spectrum for electrons will be represented by $I(E) d E$, which is the track length traveled by all electrons in the energy range $E$ to $E+d E$. $H(\alpha) d \alpha$ represents the photon spectrum similarly. ${ }^{5}$ In all cases energies are in units of $m c^{2}$, and the electron energies include the rest energy.

To set up equations in terms of $I(E)$ and $H(\alpha)$, a time-independent source is assumed. Physically, this means a continuous cascade in which the average number of electrons, positrons, and photons at any given energy remains constant with time. The equations will be derived in a general form, and considerations of the interactions and cross-sectional forms will be reserved for a separate section.

Continuity equations for $I(E)$ and $H(\alpha)$ can be written as follows:

$$
\begin{gathered}
\int_{E}^{\infty} d E^{\prime} k^{e s}\left(E^{\prime}, E\right) I\left(E^{\prime}\right)=s^{e g}(E)+\int_{E}^{\infty} d \alpha^{\prime} k^{e g}\left(\alpha^{\prime}, E\right) H\left(\alpha^{\prime}\right) \\
\int_{\alpha}^{\infty} d \alpha^{\prime} k^{p s}\left(\alpha^{\prime}, \alpha\right) H\left(\alpha^{\prime}\right)=s^{p g}(\alpha)+\int_{\alpha}^{\infty} d E^{\prime} k^{p g}\left(E^{\prime}, \alpha\right) I\left(E^{\prime}\right),
\end{gathered}
$$

where

$k^{e s}\left(E^{\prime}, E\right)$ is the total probability per unit path length traveled for an electron of energy $E^{\prime}$ to undergo an interaction that removes it from the energy range above $E$,

$k^{e g}\left(\alpha^{\prime}, E\right)$ is the total probability per unit path length traveled for a photon of energy $\alpha^{\prime}$ to generate an electron of energy greater than $E$,

$s^{e g}(E)$ is the total source strength for generating electrons with energies greater than $E$, and $k^{p s}\left(\alpha^{\prime}, \alpha\right), k^{p g}\left(E^{\prime}, \alpha\right)$, and $s^{p g}(\alpha)$ are similar quantities with the photon and electron roles reversed.

Note that the superscripts refer to "electron scattering," "photon generating," etc., although strictly speaking the interactions include types other than scattering. Physically, eq (1) states that for a time-dependent source the rate at which electrons are removed from the energy range above $E$ is equal to the rate at which electrons come into the energy range above $E$. Equation (2) has the same meaning for photons.

Equations (1) and (2) completely define $I(E)$ and $H(\alpha)$ for specified sources; however, these equations as they stand are not very suitable for numerical work. It is advantageous to make further transformations suggested by the nature of the variations of spectra and cross sections. The particular choice given here is based largely on numerical and analytic exploratory calculations that are not sufficiently pertinent to the main discussion to warrant a detailed description, but some general arguments to justify this choice are given below.

$I(E)$ may vary rapidly if $s^{e g}(E)$ is discontinuous (i. e., if the source is concentrated at some energy), as for example, in the case of figure 1 in reference [8]. A more slowly varying function on which to base a step-by-step numerical integration is

$$
F(E)=\int_{E}^{\infty} d E^{\prime} I\left(E^{\prime}\right)
$$

which gives the track length traveled by all electrons while their energy is above $E$. The function $H(\alpha)$ may also be singular if the source function $s^{p g}(\alpha)$ is discontinuous, but photon cross sections permit the removal of such singularities by iteration (see, for example, reference [9]). It is therefore not necessary to utilize an integral form of the photon spectrum.

Equation (1) could be rewritten in terms of $F(E)$ by partial integration, but this results in the differentiation of $k^{e s}\left(E^{\prime}, E\right)$, which is already singular as $E^{\prime}$ approaches $E$. A further transformation is needed to make the equation more tractable, namely integration of all terms of eq (1). With regard to eq $(2), k^{p s}\left(\alpha^{\prime}, \alpha\right)$ and $k^{p g}\left(E^{\prime}, \alpha\right)$ are integrals of nonsingular cross sections. It is therefore possible, and in fact advantageous, to accompany the partial integration of the last term in eq (2) with a differentiation of all terms in the equation.

o $(E)$ and $H(\alpha)$ are in the units $\mathrm{g} \mathrm{cm}^{-2}\left(\mathrm{Mc}^{2}\right)^{-1} \mathrm{sec}^{-1}$. These are track-length units referring to a source of one electron. The dimensions of $I(E)$ and $H(\alpha)$ change to those of flux if the source is 1 electron per gram per second. 
Applying these transformations we obtain for the left side of eq (1)

$$
\begin{aligned}
\int_{E}^{\infty} d E^{\prime} \int_{E^{\prime}}^{\infty} d E^{\prime \prime} k^{e 8}\left(E^{\prime \prime}, E^{\prime}\right) I\left(E^{\prime \prime}\right) & =\int_{E}^{\infty} d E^{\prime} I\left(E^{\prime}\right) \int_{E}^{E^{\prime}} d E^{\prime \prime} k^{e s}\left(E^{\prime}, E^{\prime \prime}\right) \\
& =\int_{E}^{\infty} d E^{\prime} F\left(E^{\prime}\right) \frac{\partial}{\partial E^{\prime}} \int_{E}^{E^{\prime}} d E^{\prime \prime} k^{e s}\left(E^{\prime}, E^{\prime \prime}\right) .
\end{aligned}
$$

The other term in the integration by parts, $-F\left(E^{\prime}\right)\left[\int_{E}^{E^{\prime}} d E^{\prime \prime} k^{e s}\left(E^{\prime} E^{\prime \prime}\right)\right]_{E}^{\infty}$, is zero at both limits.

Equation (1) thus becomes

$$
\int_{E}^{\infty} d E^{\prime} K^{e s}\left(E^{\prime}, E\right) F\left(E^{\prime}\right)=S^{e g}(E)+\int_{E}^{\infty} d \alpha^{\prime} K^{e g}\left(\alpha^{\prime}, E\right) H\left(\alpha^{\prime}\right),
$$

where

$$
\begin{aligned}
K^{e s}\left(E^{\prime}, E\right) & =\frac{\partial}{\partial E^{\prime}} \int_{E}^{E^{\prime}} d E^{\prime \prime} k^{e s}\left(E^{\prime}, E^{\prime \prime}\right), \\
K^{e g}\left(\alpha^{\prime}, E\right) & =\int_{E}^{\alpha^{\prime}} d E^{\prime} k^{e g}\left(\alpha^{\prime}, E^{\prime}\right), \\
S^{e g}(E) & =\int_{E}^{\infty} d E^{\prime} s^{e g}\left(E^{\prime}\right) .
\end{aligned}
$$

Equation (2) becomes

$$
\mu(\alpha) H(\alpha)-\int_{\alpha}^{\infty} d \alpha^{\prime} K^{p s}\left(\alpha^{\prime}, \alpha\right) H\left(\alpha^{\prime}\right)=S^{p g}(\alpha)+\int_{\alpha}^{\infty} d E^{\prime} F\left(E^{\prime}\right) K^{p g}\left(E^{\prime}, \alpha\right),
$$

Note that the other term on the right side,

$$
\frac{\partial}{\partial \alpha}\left\{\left.F\left(E^{\prime}\right) k^{p g}\left(E^{\prime}, \alpha\right)\right|_{E^{\prime}=\alpha} ^{\infty}\right\},
$$

obtained after integration by parts, is zero at both limits.

The various quantities in (4) are defined as follows:

$$
\mu(\alpha)=k^{p s}(\alpha, \alpha)
$$

is the total photon interaction probability per unit path length for photons at energy $\alpha$.

$$
K^{p s}\left(\alpha^{\prime}, \alpha\right)=\frac{\partial k^{p s}\left(\alpha^{\prime}, \alpha\right)}{\partial \alpha}=N Z \sigma\left(\alpha^{\prime}, \alpha\right),
$$

where $\sigma\left(\alpha^{\prime}, \alpha\right)$ is the photon differential scattering cross section per electron, and $N$ is the number of atoms per gram.

$$
K^{p g}\left(E^{\prime}, \alpha\right)=-\frac{\partial^{2} k^{p g}\left(E^{\prime}, \alpha\right)}{\partial E^{\prime} \partial \alpha}=\frac{\partial}{\partial E^{\prime}} N \sigma\left(E^{\prime}, \alpha\right),
$$

where $\sigma\left(E^{\prime}, \alpha\right)$ is the cross section per atom for producing radiation.

$$
S^{p g}(\alpha)=-\frac{\partial s^{p g}(\alpha)}{\partial \alpha}
$$

s the photon source strength at $\alpha$. 


\section{Interactions and Cross Sections}

Equations (3) and (4) as derived in section 4 make no reference to specific interactions or cross-sectional forms, and consequently this choice can be adapted to the specific nature of each problem. In terms of the interactions discussed in section 2 , the functions $K$ may be written

$$
\begin{aligned}
K^{e s}\left(E^{\prime}, E\right) & =K_{\text {bremsstrahlung losses }}+K_{\text {atomic interactions }}, \\
K^{e g}\left(\alpha^{\prime}, E\right) & =K_{\text {pair production }}+K_{\text {Compton electrons }}, \\
K^{p s}\left(\alpha^{\prime}, \alpha\right) & =K_{\text {Compton photons }}, \\
K^{p g}\left(E^{\prime}, \alpha\right) & =K_{\text {bremsstrablung photons }}, \\
\mu(\alpha) & =\mu(\alpha)_{\text {pair production }}+\mu(\alpha)_{\text {Compton photons }}+\mu(\alpha)_{\text {absorption }} .
\end{aligned}
$$

At this point accurate cross-sectional forms could be introduced in the equations, but an examination of their suitability can be made with simplified forms similar to whose used by earlier investigators. Specifically, (a) extreme relativistic cross sections are used, (b) complete screening is assumed, and (c) the rest mass of the electron has been neglected insofar as it influences the limits of the integrals. Detailed cross sections are given in appendix 1. The $K$ functions derived from these cross-sectional forms are

$$
\begin{aligned}
K^{e s}\left(E^{\prime}, E\right)=N 4 \bar{\varphi}\left\{\left[-\frac{1}{3}-\frac{4}{3} \ln (1-\eta)\right.\right. & \left.+\frac{4}{3}(1-\eta)-\frac{1}{6} \eta^{2}-\frac{1}{3} \eta^{3}\right] \ln 183 Z^{-1 / 3} \\
& \left.-\frac{1}{18}-\frac{1}{9} \ln (1-\eta)+\frac{1}{9}(1-\eta)-\frac{\eta^{2}}{18}\right\}+N Z \frac{3}{4} \varphi_{T} \frac{1}{E^{\prime}}\left[\frac{12 \eta-1}{(1-\eta)}\right],
\end{aligned}
$$

where $\eta=E / E^{\prime}$;

$K^{e g}\left(\alpha^{\prime}, E\right)=N 8 \bar{\varphi} \alpha^{\prime}\left\{\left[\frac{7}{18}-\frac{7}{9} \eta+\frac{1}{2} \eta^{2}-\frac{2}{9} \eta^{3}+\frac{1}{9} \eta^{4}\right] \ln 183 Z^{-1 / 3}-\frac{1}{108}+\frac{\eta}{54}-\frac{\eta^{3}}{54}\right.$

where $\eta=E / \alpha^{\prime}$;

$$
\left.+\frac{\eta^{4}}{108}\right\}+N Z \frac{3}{8} \varphi_{T}\left[(1-\eta)\left(\ln E+\ln \frac{1-\eta}{\eta}+\ln 2-\frac{1}{2}\right)+\frac{1}{2} \eta^{2}-\frac{1}{6} \eta^{3}-\frac{1}{3}\right]
$$

$$
K^{p s}\left(\alpha^{\prime}, \alpha\right)=N Z \frac{3}{8} \varphi_{T} \frac{1}{\alpha^{2}}\left(\eta^{3}+\eta\right),
$$

where $\eta=\alpha / \alpha^{\prime}$;

where $\eta=\alpha / E^{\prime} ;$ and

$$
K^{p g}\left(E^{\prime}, \alpha\right)=N 4 \bar{\varphi} \frac{1}{\alpha^{2}}\left[\left(-\frac{4}{3} \eta^{2}+2 \eta^{3}\right) \ln 183 Z^{-1 / 3}-\frac{1}{9} \eta^{2}\right]
$$

$$
\mu(\alpha)=N 4 \bar{\varphi}\left[\frac{7}{9} \ln 183 Z^{-1 / 3}-\frac{1}{54}\right]+N Z \frac{3}{8} \bar{\varphi}_{T} \frac{1}{\alpha}\left[\ln 2 \alpha+\frac{1}{2}\right]
$$

\section{Numerical Treatment of the Problem}

In determining a convenient system for the numerical integration, we note that the functions $K$ depend primarily on ratios between the two energies involved. We wish to take advantage of this scaling property by choosing integration steps of equal length in the logarithm of this energy ratio. This choice of integration mesh has the additional advantages that one can integrate over an enormous energy range with comparatively few steps, and that a crosssectional tabulation can be made that is applicable to widely different energy ranges.

The numerical integration is performed by Simpson's rule, using modifications of the familiar $\left(\frac{1}{3}, \frac{4}{3}, \frac{2}{3}, \frac{4}{3}, \frac{1}{3}\right) \Delta$ set of weights to make the rule apply also to an odd number of intervals. 
The integrals were computed essentially as follows:

$$
\begin{aligned}
\int d E^{\prime} K^{e s}\left(E^{\prime}, E\right) F\left(E^{\prime}\right) & =\int d\left(\ln E^{\prime}\right) E^{\prime} K^{e s}\left(E^{\prime}, E\right) F\left(E^{\prime}\right) \\
& =\sum_{i=0}^{n} \omega^{\prime}{ }_{i n} E_{i} K^{e s}\left(E_{i}, E_{n}\right) F\left(E_{i}\right) \\
& =\sum_{i=0}^{n} \omega_{i n} K^{e s}\left(E_{i}, E_{n}\right) F\left(E_{i}\right) .
\end{aligned}
$$

Note that the integration weights $\omega_{i n}$ decrease in size as the energy decreases. More details are given in appendix 2 .

Despite the transformations discussed in section 4 , the function $K^{e s}\left(E^{\prime}, E\right)$ in eq (3) is still singular. However, this singularity causes no difficulty if one writes the integral in the following manner:

$$
\int_{E}^{\infty} d E^{\prime} K^{e s}\left(E^{\prime}, E\right) F\left(E^{\prime}\right)=\int_{E}^{\infty} d E^{\prime} K^{e s}\left(E^{\prime}, E\right)\left[F\left(E^{\prime}\right)-F(E)\right]+F(E) \int_{E}^{\infty} d E^{\prime} K^{e s}\left(E^{\prime}, E\right) .
$$

Note that the first term on the right is no longer singular and can be integrated numerically. The last term can be evaluated without difficulty by integrating the singular parts of $K^{e s}\left(E^{\prime}, E\right)$ analytically. Notice also that as $E^{\prime} \rightarrow E$, the first integrand is finite, approaching the limit $A[\partial F(E) / \partial E]$, where $A=\lim _{E^{\prime} \rightarrow E}\left(E^{\prime}-E\right) K^{e s}\left(E^{\prime}, E\right)$. This term is very small and needs to be evaluated only approximately. A three-point numerical differentiation formula, described in appendix 2, was adopted for this purpose, the corresponding weights being denoted by $a_{i}$. By utilizing these devices, we represent the first integral of eq (3) as the sum of terms

$$
F\left(E_{n}\right)\left[\mathscr{K}^{e s}\left(E_{n}\right)-\sum_{i=0}^{n-1} \omega_{i n} K^{e s}\left(E_{i}, E_{n}\right)+A a_{n}\right]+\sum_{i=0}^{n-1}\left[\omega_{i n} K^{e s}\left(E_{i}, E_{n}\right)+A a_{i}\right] F\left(E_{i}\right),
$$

where $\mathscr{K}^{e s}(E)=\int_{E}^{\infty} d E^{\prime} K^{e s}\left(E^{\prime}, E\right)$. Note that $a_{i}$ is zero except for $i=n, n-1, n-2$.

If the functional form of $K^{e s}\left(E^{\prime}, E\right)$ is that given in section 5 ,

$$
\begin{aligned}
\mathscr{K}^{e s}(E)=N 4 \bar{\varphi} E_{0}\left\{\left[-\frac{1}{3}-\frac{4}{3}(1-\eta) \ln (1-\eta)+\frac{4}{3}(1-\eta)+\frac{1}{6} \eta^{2}+\frac{1}{6} \eta^{3}\right] \ln 183 Z^{-1 / 3}-\frac{1}{18}\right. \\
\left.\quad-\frac{1}{9}(1-\eta) \ln (1-\eta)+\frac{1}{9}(1-\eta)+\frac{\eta^{2}}{18}\right\}+N Z_{\frac{3}{4} \varphi_{T}} \ln \frac{\eta(1-\eta) E_{0}}{\bar{Q}}
\end{aligned}
$$

where $\eta=E / E_{0}$, and $E_{0}$ is the highest energy of the source of electrons and photons. For a discussion of the choice of the cutoff value $\bar{Q}$, see reference [8]. In this paper it has been evaluated as follows:

$$
\bar{Q}=\frac{1}{2}\left(\frac{I_{0} Z}{m c^{2}}\right)^{2} \bar{E}_{0}^{2} \frac{e}{-1}=4.9214 \times 10^{-11}
$$

where $I_{0}=13.5 \mathrm{ev}$.

The function $K^{p g}\left(E^{\prime}, \alpha\right)$ in eq (4) is not singular, but the exact expression varies rapidly as $E^{\prime} \rightarrow \alpha$. This can easily be taken into account by using addition-subtraction as before:

$$
\int_{\alpha}^{\infty} d E^{\prime} K^{p g}\left(E^{\prime}, \alpha\right) F\left(E^{\prime}\right)=\int_{\alpha}^{\infty} d E^{\prime} K^{p g}\left(E^{\prime}, \alpha\right)\left[F\left(E^{\prime}\right)-F(E)_{E=\alpha}\right]+F(E)_{E=\alpha} \int_{\alpha}^{\infty} d E^{\prime} K^{p g}\left(E^{\prime}, \alpha\right) .
$$


Written as a sum, this term becomes

$$
F\left(E_{n}\right)\left[\mathscr{K}^{p g}\left(\alpha_{n}\right)-\sum_{i=0}^{n-1} \omega_{i n} K^{p g}\left(E_{i}, \alpha_{n}\right)\right]+\sum_{i=0}^{n-1} \omega_{i n} K^{p g}\left(E_{i}, \alpha_{n}\right) F\left(E_{i}\right)
$$

where $\mathscr{K}^{p g}(\alpha)=\int_{\alpha}^{\infty} d E^{\prime} K^{p g}\left(E^{\prime}, \alpha\right)$. If the functional form of $K^{p g}\left(E^{\prime}, \alpha\right)$ is that given in section 5 ,

$$
\mathscr{K}^{p g}(\alpha)=N 4 \bar{\varphi}_{\alpha}^{-1}\left[\left(\frac{4}{3}-\frac{4}{3} \eta+\eta^{2}\right) \ln 183 Z^{-1 / 3}+\frac{1-\eta}{9}\right],
$$

where $\eta=\alpha / E_{0}$.

Equations (3) and (4) can now be written in their final form for numerical evaluation:

$$
\begin{gathered}
F\left(E_{n}\right)=\frac{1}{C^{e}\left(E_{n}\right)}\left[-\sum_{i=0}^{n-1} W_{i n}^{e s} F\left(E_{i}\right)+\sum_{i=0}^{n-1} W_{i n}^{e g} H\left(\alpha_{i}\right)+S^{e g}\left(E_{n}\right)\right] \\
H\left(\alpha_{n}\right)=\frac{1}{C^{p}\left(\alpha_{n}\right)}\left[F\left(E_{n}\right) D^{p g}\left(\alpha_{n}\right)+\sum_{i=0}^{n-1} W_{i n}^{p g} F\left(E_{i}\right)+\sum_{i=0}^{n-1} W_{i n}^{p s} H\left(\alpha_{i}\right)+S^{p g}\left(\alpha_{n}\right)\right],
\end{gathered}
$$

where

$$
\left.\begin{array}{rl}
W_{i n}^{e s} & =\omega_{i n} K^{e s}\left(E_{i}, E_{n}\right)+A a_{i}, \\
W_{i n}^{e g} & =\omega_{i n} K^{e g}\left(\alpha_{i}, E_{n}\right), \\
W_{i n}^{p g} & =\omega_{i n} K^{p g}\left(E_{i}, \alpha_{n}\right), \\
W_{i n}^{p s} & =\omega_{i n} K^{p s}\left(\alpha_{i}, \alpha_{n}\right), \\
C^{e}\left(E_{n}\right) & =\mathscr{K}^{e s}\left(E_{n}\right)-\sum_{i=0}^{n-1} \omega_{i n} K^{e s}\left(E_{i}, E_{n}\right)+A a_{n}, \\
C^{p}\left(\alpha_{n}\right) & =\mu\left(\alpha_{n}\right)-\omega_{n n} K^{i s}\left(\alpha_{n}, \alpha_{n}\right), \\
D^{p g}\left(\alpha_{n}\right) & =\mathscr{K}^{p g}\left(\alpha_{n}\right)-\sum_{i=0}^{n-1} W_{i n}^{p g} .
\end{array}\right\}
$$

These seven functions depend only on cross-sectional forms, and they are evaluated separately prior to the main calculation. Note that eq (5) depends on $F\left(E_{i}\right)$ and $H\left(\alpha_{i}\right)$ but not on $H\left(\alpha_{n}\right)$, while eq (6) depends on $F\left(E_{n}\right)$ and on $F\left(E_{i}\right)$ and $H\left(\alpha_{i}\right)$. Therefore, eq (6) must be determined after eq (5) at each step in the integration. The problem of how to start the calculation is taken up in the next section.

\section{Special Solution Near $E_{0}$}

$F(E)$ and $H(\alpha)$ must vanish at the highest energy, $E_{0}$. It would be possible to use eqs (5) and (6), with the cross-sectional forms that have been listed, to solve for the spectra near $E_{0}$. But it was found more convenient and accurate to do a separate calculation because the solution near $E_{0}$ has certain special features. In particular, we note that (1) for small energy losses it is always possible to use extremely simple cross-sectional forms, (2) the linkage between the electron and photon equations can be simplified because the higher cascades have negligible influence in this energy region, and (3) it is possible, though not necessarily desirable, to seek analy tic solutions.

Because even an analytic development leads to a numerical integration, it appeared most expeditious to employ eq (3), with the simplifications just mentioned and a finer integration spacing, to develop a solution in this region. In particular, for an electron-generated cascade, the term for generating electrons from photons and the term $A[\partial F(E) / \partial E]$ are very small and can be neglected. The remaining functions $K$ can be written in the approximate form: 


$$
\begin{aligned}
K^{e s}\left(E^{\prime}, E\right) & =\frac{A}{E^{\prime}-E^{\prime}}-B \ln \frac{E^{\prime}-E}{E_{0}}+C, \\
\mathscr{K}^{e s}(E) & =A \ln \frac{E_{0}-E}{Q}-B\left(E_{0}-E\right) \ln \frac{E_{0}-E}{e E_{0}}+C\left(E_{0}-E\right),
\end{aligned}
$$

where $A, B$, and $C$ are constants (see appendix 1 ).

The quantities (7) were tabulated at $E_{0} \times 2^{-m / 6}$, where $m=\frac{1}{8}, \frac{1}{4}, \frac{1}{2}, 1$. This spacing required a special set of weights; but otherwise the evaluation in this special case is carried out by using the appropriate form of eq (5) in a manner similar to the main calculation. Results of this special solution not only give the solution near $E_{0}$ but also permit a more accurate evaluation of the integrals in eq (3) for $\alpha^{\prime}, E^{\prime} \geq E_{0} \times 2^{-1 / 6}$. In this energy region the use of Simpson's rule with $2^{-1 / 6}$ spacing is not really a good enough approximation because $F(E)$ has large nonquadratic variations.

\section{Calculations}

The calculations in this paper are based on a monoenergetic source of 1 electron per second at $360 m c^{2}$ in lead. Results were first obtained for the special solution near $E_{0}$. The main calculation, extending down te $5.6 m c^{2}$, was carried out in two parts, using the mesh $E_{0} \times 2^{-m / 6}$ to the energy $\frac{1}{4} E_{0}$ and the mesh $E_{0} \times 2^{-m / 3}$ from that energy to $E=5.6 m c^{2}$.
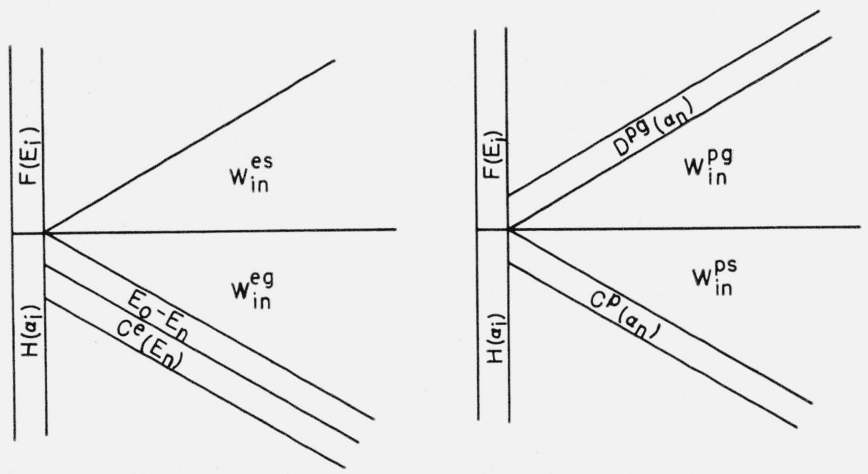

Figure 1. Arrangement of tabulated functions.

The calculation was facilitated by arranging a triangular tabulation of the four $W$ 's and a linear tabulation of the $C$ 's and $D$ in a form shown in figure 1 . Most of the work went into tabulating $K$ functions and $W$ 's, the final computation being carried out in a few hours. The results are given in table 1.

Any error had the effect of introducing an oscillation in the final computation, with alternate points being too high and too low. The integration procedure appeared stable in that such oscillations tended to damp out slowly rather than to magnify. By plotting the results it was possible to use these oscillations to find errors. The final sequence of numbers is smooth, i. e., nonoscillatory, to better than 1 percent, which may indicate that the integration procedure introduced errors no larger than this, although the possibility that errors may have piled up at low energy is not excluded.

\begin{tabular}{|c|c|c|c|c|c|c|c|c|}
\hline$E$ & $F(E)$ & $H(\alpha)$ & $E$ & $F(E)$ & $H(\alpha)$ & $E$ & $F(E)$ & $H(\alpha)$ \\
\hline $\begin{array}{l}360.0 \\
354.8 \\
349.8 \\
339.8 \\
320.7\end{array}$ & $\begin{array}{l}0 \\
0.751 \\
.980 \\
\text { 1. } 35 \\
1.76\end{array}$ & $\begin{array}{l}0 \\
0.00488 \\
.00652\end{array}$ & $\begin{array}{l}160.4 \\
142.9 \\
127.3 \\
113.4 \\
101.0\end{array}$ & $\begin{array}{l}5.26 \\
5.92 \\
6.52 \\
7.31 \\
7.97\end{array}$ & $\begin{array}{l}0.0409 \\
.0537 \\
.0693 \\
.0901 \\
.115\end{array}$ & $\begin{array}{l}28.4 \\
22.5 \\
17.9 \\
14.2 \\
11.3\end{array}$ & $\begin{array}{l}22.4 \\
26.8 \\
31.4 \\
36.5 \\
41.6\end{array}$ & $\begin{array}{l}\text { 1. } 44 \\
\text { 2. } 20 \\
\text { 3. } 27 \\
4.80 \\
6.85\end{array}$ \\
\hline $\begin{array}{l}285.7 \\
254.6 \\
226.8 \\
202.0 \\
180.0\end{array}$ & $\begin{array}{l}2.47 \\
3.05 \\
3.61 \\
4.14 \\
4.71\end{array}$ & $\begin{array}{l}.00985 \\
.0135 \\
.0180 \\
.0238 \\
.0314\end{array}$ & $\begin{array}{l}90.0 \\
71.4 \\
56.7 \\
45.0 \\
35.7\end{array}$ & $\begin{array}{l}8.91 \\
10.5 \\
13.0 \\
15.6 \\
18.9\end{array}$ & $\begin{array}{l}.148 \\
.235 \\
.384 \\
.601 \\
.944\end{array}$ & $\begin{array}{l}\text { 8. } 93 \\
7.09 \\
5.63\end{array}$ & $\begin{array}{l}47.9 \\
53.8 \\
60.3\end{array}$ & $\begin{array}{l}10.1 \\
13.8 \\
19.2\end{array}$ \\
\hline
\end{tabular}

TABLE 1. Results 


\section{Discussion of Results}

The results in table 1 are plotted in figures 2 and 3 , where they are compared with the results of Richards and Nordheim [6] and calculations based on the analytic solution given by Rossi and Greisen [7]. (See appendix 3 for a discussion of the normalization.) For the comparatively low initial energy considered here, the equations of Rossi and Greisen are only accurate in a very limited energy region around $130 m c^{2}$. At lower energies, as pointed out by Richards and Nordheim [6], a more accurate representation of the interaction probabilities is necessary. The Richards and Nordheim numerical calculation was based on more accurate cross-sectional forms, but their results are normalized to those of Rossi and Greisen at $131 m c^{2}$. Thus, near that energy, the significant comparison is with Rossi and Greisen. At lower energies, due to differences in the cross-sectional functions involved, the three curves go apart. At energies near $E_{0}$ no comparable calculations have been found. Thus the only energy region where close agreement between the spectra calculated in this paper and those given by the other authors could be expected is from $\frac{1}{2}$ to $\frac{1}{4} E_{0}$. In this region the agreement is shown in figures 2 and 3 to be quite good.

Through a consideration of the specific cross-sectional forms used in the three calculations, some further comparisons are possible at lower energies. For example, the spectra of Rossi and Greisen indicate a larger number of electrons and a smaller number of photons than reported in this paper. This is caused mainly by the cross section for pair production, which is significantly lower under the complete screening approximation than the asymptotic form used by Rossi and Greisen. Thus, in the calculations in this paper, low-energy photons do not generate as many electrons, and there results a decrease in $F(E)$ and an increase in $H(\alpha)$ relative to Rossi-Greisen. The spectra of Richards and Nordheim indicate a much larger number of electrons and photons at low energies. Although this must be traceable similarly to a difference in cross sections, the reasoning is not as straightforward in this case.

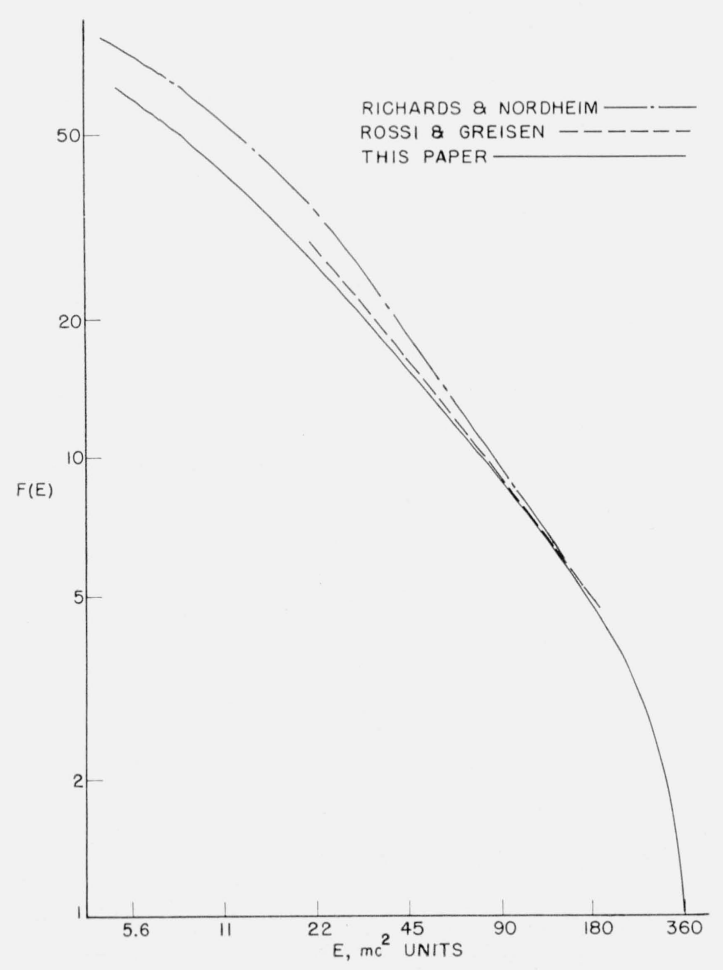

Figure 2. Comparison of $F(E)$ with previous results.

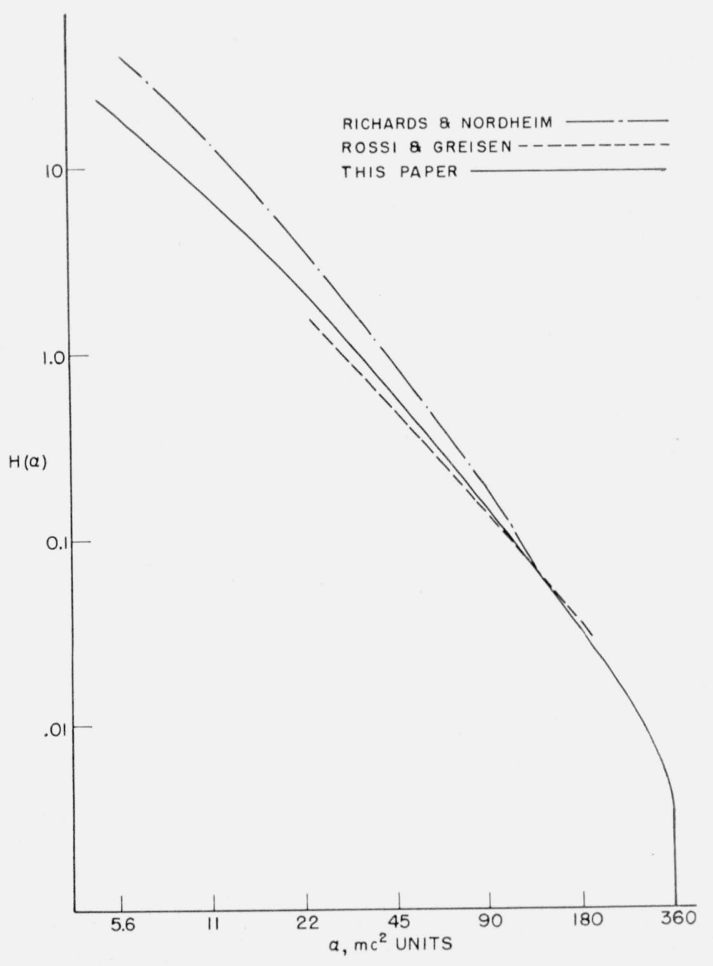

FiguRe 3. Comparison of $H(\alpha)$ with previous results. 


\section{Final Comments}

The agreement as to general trends and as to absolute magnitude near $\frac{1}{2} E_{0}$ demonstrates the feasibility of the calculational procedure developed in this paper. Logically, the next step is to introduce accurate cross-sectional forms into the equations to obtain more accurate spectra. Alternatively, one could move immediately toward a solution of the spatial and directional distributions. This would involve computing the higher moments in the linked set of equations of which these spectra form the zeroth moment (for an example of this, see reference [10]).

\section{Appendixes}

\section{Appendix 1. Cross-Sectional Forms}

The following detailed cross-sectional forms were used to derive the functions $K$ discussed in section 5 .

Bremsstrahlung losses [11]:

$$
\sigma_{b}\left(E^{\prime}, E\right)=4 \bar{\varphi} \frac{1}{E^{\prime}}\left(\frac{\eta}{1-\eta}\right)\left[\left(\frac{1+\eta^{2}}{\eta}-\frac{2}{3}\right) \ln 183 Z^{-1 / 3}+\frac{1}{9}\right],
$$

where $\eta=E / E^{\prime}$.

Electron atomic interactions [8]:

$$
\sigma_{M}\left(E^{\prime}, E\right)=\frac{3}{4} \varphi_{T} \frac{1}{E^{\prime 2}}\left[\frac{1}{(1-\eta)^{2}}+\frac{1}{\eta^{2}}\right]
$$

where $\eta=E / E^{\prime}$.

Pair production (either electron or positron) [11]:

$$
\sigma_{p p}\left(\alpha^{\prime}, E\right)=8 \bar{\varphi} \frac{1}{\alpha^{\prime}}\left[\left(\frac{4}{3} \eta^{2}-\frac{4}{3} \eta+1\right) \ln 183 Z^{-1 / 3}-\frac{1}{9} \eta(1-\eta)\right]
$$

where $\eta=E / \alpha^{\prime}$.

Compton electrons:

$$
\sigma_{c e}\left(\alpha^{\prime}, E\right)=\frac{3}{8} \varphi_{T} \frac{1}{\alpha^{\prime 2}}\left[\frac{\alpha^{\prime}}{\alpha^{\prime}-E+1}+\frac{\alpha^{\prime}-E+1}{\alpha^{\prime}}\right] .
$$

Compton photons:

$$
\sigma_{c}\left(\alpha^{\prime}, \alpha\right)=\frac{3}{8} \varphi_{T} \frac{1}{\alpha^{\prime 2}}\left[\frac{\eta^{2}+1}{\eta}\right]
$$

where $\eta=\alpha / \alpha^{\prime}$.

Bremsstrahlung pbotons [11]:

$$
\sigma_{b p}\left(E^{\prime}, \alpha\right)=4 \bar{\varphi} \frac{1}{\alpha}\left[\left(\frac{4}{3}-\frac{4}{3} \eta+\eta^{2}\right) \ln 183 Z^{-1 / 3}+\frac{1-\eta}{9}\right],
$$

where $\eta=\alpha / E^{\prime}$.

The cross-sectional constants were evaluated as follows:

$$
\varphi=\frac{Z^{2} r_{0}^{2}}{137} \frac{Z+1}{Z}=3.9435 \text { barns }, \quad Z=82 ;
$$

and

$$
\varphi_{T}=\frac{8}{3} \pi r_{0}^{2}=0.6652 \text { barn }
$$

The constants $A, B$, and $C$ in the special solution near $E_{0}$ (section 7 ) are obtained from the expression for $K^{e s}\left(E^{\prime}, E\right)$; they are coefficients of the terms proportional to $F^{-1}(1-\eta)^{-1}$, $-\ln (1-\eta)$, and 1 , respectively, in the limit $E, E^{\prime} \rightarrow E_{0}$ : 


$$
\begin{aligned}
& A=N Z \frac{3}{4} \varphi_{T}=0.1189 \\
& B=N 4 \bar{\varphi}\left[\frac{4}{3} \ln 183 Z^{-1 / 3}+\frac{1}{9}\right]=0.2339 \\
& C=N 4 \bar{\varphi}\left[-\frac{5}{6} \ln 183 Z^{-1 / 3}-\frac{1}{9}\right]-\frac{3}{4} N Z_{\varphi_{T}} \frac{2}{E_{0}}=-0.1487 .
\end{aligned}
$$

\section{Appendix 2. Integration and Differentiation Weights}

To obtain the integration weights $\omega_{i n}$ used in connection with eqs (5) and (6), the following formulas were used:

$$
\begin{aligned}
& \int_{x_{0}-\Delta}^{x_{0}+\Delta} d x f(x) \approx \frac{\Delta}{3}\left[f\left(x_{0}+\Delta\right)+4 f\left(x_{0}\right)+f\left(x_{0}-\Delta\right)\right] \\
& \int_{x_{0}}^{x_{0}+\Delta} d x f(x) \approx \frac{\Delta}{12}\left[5 f\left(x_{0}+\Delta\right)+8 f\left(x_{0}\right)-f\left(x_{0}-\Delta\right)\right], \\
& \int_{x_{0}-\Delta}^{x_{0}+2 \Delta} d x f(x) \approx \frac{3 \Delta}{4}\left[f\left(x_{0}+2 \Delta\right)+3 f\left(x_{0}\right)\right],
\end{aligned}
$$

where $f(x)$ is a function that varies slowly for $x$ in the region of integration.

\begin{tabular}{|c|c|c|}
\hline$E$ & $F(E)=69.86 F_{R N}(E)$ & $H(\alpha)=5.329 g(K)$ \\
\hline $\begin{array}{r}131 \\
91.8 \\
65.6 \\
39.3 \\
26.2 \\
19.7 \\
13.1 \\
6.56\end{array}$ & $\begin{array}{r}6.43 \\
9.08 \\
12.6 \\
21.0 \\
30.0 \\
37.7 \\
49.6 \\
71.3\end{array}$ & $\begin{array}{c}0.0639 \\
.171 \\
.384 \\
1.12 \\
2.61 \\
4.74 \\
10.7 \\
34.1\end{array}$ \\
\hline
\end{tabular}

For differentiation the weights $a_{i}$ were determined by using the following formula:

$$
\frac{\partial F(n)}{\partial E_{n}}=F\left(E_{n}\right)\left[\sum_{i \neq n} \frac{1}{E_{n}-E_{i}}\right]+\sum_{i \neq n} F\left(E_{i}\right)\left[\frac{\prod_{j \neq i}\left(E_{n}-E_{j}\right)}{\prod_{j \neq i}\left(E_{i}-E_{j}\right)}\right] .
$$

\section{Appendix 3. Normalization of Previous Results}

The following results were obtained by using a table of calculations given by Richards and Nordheim [6].

The normalization factors arise in the following manner:

$$
\begin{aligned}
& 69.86=\left[0.437 \times \frac{184}{6.7}\right] \times \text { density of lead }(11.005) \times \text { radiation length }(0.529) \\
& 5.329=69.86 \times \text { energy conversion factor } \frac{1}{13.11} .
\end{aligned}
$$

For a discussion of the factor in square brackets, see reference [6]. 
The following results were obtained by using the equations of Rossi and Greisen [7] under "approximation B":

\begin{tabular}{|c|c|c|}
\hline$E$ & $F(E)=5.822 Z_{\pi}\left(E_{0}, E\right)$ & $H(\alpha)=2.975 z_{\gamma}\left(E_{0}, W\right)$ \\
\cline { 2 - 3 } & & \\
180 & 4.77 & 0.0348 \\
90 & 9.02 & .132 \\
45 & 16.4 & .482 \\
22.5 & 28.5 & 1.70 \\
\hline
\end{tabular}

The normalization factors arise in the following manner:

$$
\begin{aligned}
& 5.822=\text { density of lead }(11.005) \times \text { radiation length }(0.529), \\
& 2.975=5.822 \times m c^{2}(0.511) .
\end{aligned}
$$

\section{References}

[1] H. Brysk, Phys. Rev. 96, 419 (1954).

[2] B. Rossi, High energy particles, chap. 5 (Prentice-Hall, Inc., New York, N. Y., 1952).

[3] N. Arley, Proc. Roy. Soc. (London) [A] 168, 519 (1938).

[4] I. Tamm and S. Belenky, J. Phys. USSR 1, 177 (1939); Phys. Rev. 70, 660 (1946).

[5] F. L. Friedman, Tech. Rept. 31, Laboratory for Nuclear Science and Engineering, MIT (1949).

[6] J. A. Richards and L. W. Nordheim, Phys. Rev. 74, 1106 (1948).

[7] B. Rossi and K. Greisen, Rev. Mod. Phys. 13, 240 (1941).

[8] L. V. Spencer and U. Fano, Phys. Rev. 93, 1172 (1954)

[9] U. Fano, L. V. Spencer, and M. Berger, Penetration and diffusion of X-rays, Handbuch der Physik (to be published).

[10] L. V. Spencer and U. Fano, J. Research NBS 46, 446 (1951) RP2213.

[11] W. Heitler, The quantum theory of radiation, 2d ed. (Oxford Univ. Press, London, 1944).

Washington, May 15, 1957. 